\title{
26 Research Square \\ The role of Bacillus acidophilus in osteoporosis and its roles in osteocyte proliferation and differentiation
}

\section{Chen Chen}

Tianjin Medical University General Hospital

\section{Baokang Dong}

Tianjin first hospital

\section{Yuming Wang}

Tianjin Medical University General Hospital

Qiang Zhang

Tianjin Medical University General Hospital

\section{Bangmao Wang}

Tianjin Medical University General Hospital

\section{Shuzhi Feng}

Tianjin Medical University

\section{Yu Zhu ( $\nabla$ zhuyutj@126.com )}

Tianjin Huanhu Hospital https://orcid.org/0000-0003-0499-0235

\section{Research article}

Keywords: Gut Microbiota, Osteoporosis, Osteoclasts, Osteoblasts

Posted Date: September 23rd, 2019

DOI: https://doi.org/10.21203/rs.2.14816/v1

License: (c) (1) This work is licensed under a Creative Commons Attribution 4.0 International License. Read Full License 


\section{Abstract}

The microbes play an important role to physiological activities of human. Osteoporosis is one of the most closely relationship diseases with the elderly. In recent years, the studies found that gut microbiota can cause osteoporosis. These microbes may affect activity of osteoclasts and osteoblasts to affect bone metabolism. We selected 5 health people and 10 osteoporosis patients and found that the diversity of gut microbiota in osteoporosis patients are decreased and imbalance with lower abundance of lactobacillus and butylic acid bacteria, meanwhile, 25-hydroxyitamin D and procollagen type I N-terminal peptide (PINP) of osteoporosis patient was significantly lower than the normal group. The proliferation, differentiation and maturity of osteoblasts MC3T3-E1 cells was stimulated, the activity of alkaline phosphatase, concentration of osteocalcin and the expression of RUNX2, WNT2 and CTNNB1 was improved by supernatant of lactobacillus acidophilus and butanoic acids, however, the proliferation, differentiation and maturity, the expression of RANK,WNT2 and CTNNB1 in osteoclasts RAW 264.7 cells was suppressed. There is not a similar significant effect by lactobacillus rhamnosus treatment.

\section{Background}

As a mammal, humans have an incredibly large number of microbes living on and inside our bodies and a complex community of symbiotic bacteria (1). These microorganisms play an important role in the physiological activities of the human body. The largest microecosystem in the human body is the intestinal tract (2).

In recent years, increasing evidence has accumulated suggesting that the human gut microbiota can be thought of as a very important organ acquired after birth (3).New attention has been directed at the relationship between the gut microbiota and human health. The interaction between the gut microbiota and the host is mutualistic, as the host can provide a suitable environment and nutrients to support bacterial growth while the gut microbiota plays important roles in food digestion, the production of vitamins and other nutrients, and increasing resistance against invasion by foreign pathogens (4-5).In addition to these important roles, the microbiota can also affect many physiological functions of the human body. Under normal circumstances, the intestinal microecosystem remains relatively stable, and this balance is beneficial to the human body. However, a variety of factors (such as drugs, alcoholism, mental factors, gastrointestinal surgery, radiation therapy and aging) can alter this balance, resulting in the development of a variety of diseases, such as multiple enteritis, diabetes, asthma, obesity, osteoporosis and metabolic syndrome (6-10).

Osteoporosis is a disease intimately linked to aging (11).It is a common bone disease characterized by decreased bone mass and bone degeneration. Recent studies have shown that the gut microbiota is associated with decreased bone mass and the pathogenesis of osteoporosis (12). These microorganisms may alter the relative activities of osteoclasts and osteoblasts through different pathways, such as metabolite production and altering of the host metabolism and immune system, both of which can affect bone metabolism. 
Therefore, the goals of this study was to analyze the changes in the gut microbiota in osteoporosis patients and to study the effects of the metabolites of two probiotics, Lactobacillus acidophilus and Lactobacillus rhamnosus (LGG), on an osteoblast precursor cell line (MC3T3-E1) and on an osteoclast precursor cell line (RAW 264.7). By studying the relationship between the gut microbiota and osteoporosis and investigating the mechanism by which the gut microbiota affects bone metabolism from the perspective of osteoblast-mediated bone formation and osteoclast-mediated bone resorption, this study could lay a theoretical foundation for further investigation into these topics and provide data to support the use of probiotics as a clinical intervention for osteoporosis.

\section{Methods}

\subsection{Research subjects}

\subsubsection{Selection of research subjects}

10 patients with osteoporosis and osteoporotic fractures who were hospitalized in the Department of Geriatrics of General Hospital of Tianjin Medical University from January 2017 to December 2017 were randomly recruited into the osteoporosis group. 5 health people without osteoporosis were randomly recruited for the control group. The general data of normal and osteoporosis group was shown in Table1. All participants in the experiments were matched for age and gender. This study was approved by the medical ethics committee of Tianjin Medical University General Hospital on 2015 according to the Declaration of Helsinki, and patients provided informed consent before the experiments.

The inclusion criteria for the osteoporosis group included (1) patients who were diagnosed with osteoporosis by dual-energy $\mathrm{x}$-ray absorptiometry or with osteoporotic fractures by imaging and (2) nonviolent fractures.

The exclusion criteria were as follows: (1) patients with fractures caused by violence or trauma and (2) patients with other bone diseases, such as osteomalacia, renal osteodystrophy or other metabolic bone diseases or bone tumors.

\subsubsection{Analysis of the demographics data of the selected subjects}

The sex, age, height, weight and blood pressure of each selected patient were collected to calculate the mean age, mean body mass index (BMI), mean systolic blood pressure (SBP) and mean diastolic blood pressure (DBP). Fasting blood samples were obtained to test the serum levels of total protein (TP), albumin (ALB), alanine aminotransferase (ALT), aspartate aminotransferase (AST), alkaline phosphatase (ALP), serum creatinine (CREA), blood Urea (UREA), blood uric acid (UA), fasting blood glucose (FPG), 
blood calcium (Ca), blood phosphorus (P), blood potassium (K), blood sodium (Na), triglycerides $(\mathrm{TG})$, total cholesterol (TC), low-density lipoprotein cholesterol (LDL), 25-hydroxyvitamin D, osteocalcin (OC), parathyroid hormone (PTH), procollagen type I N-terminal peptide (PINP) and C-terminal telopeptide of type I collagen (CTX).

\subsubsection{Measurement of bone density and diagnosis of osteoporosis}

The medical staff of the Bone Densitometry Section of the Department of Geriatrics at the General Hospital of Tianjin Medical University used dual-energy X-ray absorptiometry (Lunar DPX Prodigy, GE Healthcare, USA) to measure the bone density of the $2^{\text {nd }}$ to the $5^{\text {th }}$ lumbar vertebrae (L2-L4) and the hip (left femoral neck, trochanter and Ward's triangle) of the patients in each group. The total bone density $\left(\mathrm{g} / \mathrm{cm}^{2}\right)$ was calculated to obtain T-Scores. The diagnostic criteria for osteoporosis were based on TScores $\leq-2.5$ in any of the following sites: lumbar vertebrae, femoral neck or total hip.

\subsection{Fecal DNA extraction and testing}

Stool specimens were collected from the patients in the osteoporosis and healthy control groups using a sterile specimen container. Approximately $1 \mathrm{gram}$ of feces was placed in the sterile stool specimen container, and the container was immediately sealed and stored in liquid nitrogen. The samples were sent to the laboratory within 1 hour for storage in a $-80^{\circ} \mathrm{C}$ freezer. A cador Pathogen 96 QIAcube HT Kit (QIAGEN, USA) was used to extract bacterial genomic DNA from the stool samples. The concentrations of the genomic DNA samples (in $2 \mu \mathrm{l}$ ) were measured with an ultraviolet-visible spectrophotometer. Pure DNA should exhibit an obvious absorption peak at an optical density of $260 \mathrm{~nm}\left(\mathrm{OD}_{260}\right)$ with an $\mathrm{OD}_{260} / \mathrm{OD}_{280}$ ratio of approximately 1.8 .

Construction of a high-throughput sequencing library and Illumina-based sequencing using a MiSeq instrument were performed by GENEWIZ (Suzhou, China). The sequencing library was constructed using the MetaVx ${ }^{T M}$ library construction kit (GENEWIZ, Inc., South Plainfield, NJ, USA). Custom polymerase chain reaction (PCR) primers were used to amplify two highly variable regions of the bacterial 16S rDNA gene (the V3 and V4 regions) using 30-50 ng of DNA as the template. The V3 and V4 regions were amplified using a forward primer with the sequence "CCTACGGRRBGCASCAGKVRVGAAT" and a reverse primer with the sequence "GGACTACNVGGGTWTCTAATCC". Library quality was evaluated using and Agilent 2100 Bioanalyzer (Agilent Technologies, Palo Alto, Calif., USA), and the library concentrations were determined using a Qubit 2.0 Fluorometer (Invitrogen, Carlsbad, CA). After the DNA library was mixed, double-end sequencing (PE, $2 \times 300$ bp) was performed using an Illumina MiSeq (Illumina, San Diego, CA, USA) instrument according to manufacturer's instructions, and the sequence data were analyzed with MiSeq Control Software (MCS) provided by MiSeq. 


\subsection{Cell culture}

Mice osteoblasts MC3T3-E1 were purchased from the Tianjin institute of orthopaedics and tranmatology and cultured in a-MEM with 10\% fetal bovine serum (Gibco; Thermo Fisher Scientifc, Inc., Waltham, MA,USA) at $37^{\circ} \mathrm{C}$ and $5 \% \mathrm{CO}_{2}$. Mice osteoclasts RAW 264.7 were obtained from the Tianjin Medical University and cultured in DMEM (High Glucose) with $10 \%$ fetal bovine serum (Gibco; Thermo Fisher Scientifc, Inc., Waltham, MA,USA), penicillin $(100 \mathrm{U} / \mathrm{ml})$ and streptomycin $(100 \mathrm{mg} / \mathrm{ml})$ at $37^{\circ} \mathrm{C}$ and $5 \%$ $\mathrm{CO}_{2}$.

\subsection{Bacterial culture and supernatant extraction}

Cryogenic vials containing the lactobacillus acidophilus (LABS)and Lactobacillus rhamnosus (LGG) solution stored in a liquid nitrogen tank were removed and completely thawed at room temperature. One milliliter of the bacterial solution was pipetted into $100 \mathrm{ml}$ of the prepared deMan, Rogosa and Sharpe (MRS) medium in a sterile laminar flow work bench, and the inoculated culture dishes were incubated at $37^{\circ} \mathrm{C}$. After 72 hours, the bacterial growth was stable and the subsequent experiments were carried out. Culture medium containing LABS andLGG was transferred to a centrifuge tube and centrifuged in a highspeed refrigerated centrifuge at $4000 \mathrm{r} / \mathrm{min}$ for $10 \mathrm{~min}$ at $4{ }^{\circ} \mathrm{C}$. After centrifugation, the supernatant was filtered through a membrane $(0.22 \mu \mathrm{m}) 3$ times to obtain bacterial supernatant.

\subsection{Osteoblast and osteoclasts proliferation}

MC3T3-E1 and RAW 264.7 cells $\left(2.5 \times 10^{3}\right.$ per well) treated with bacterial culture supernatant (mixed at ratios of $1: 20,1: 50$ and $1: 100)$ or sodium butyrate $(0.5 \mathrm{mM})$ were inoculated in 96-well plates and incubated for $48 \mathrm{~h}$. Then $20 \mu \mathrm{L}$ of tetrazolium dye (MTT) solution $(5 \mathrm{mg} / \mathrm{mL})$ was added to each well, and the plates were incubated at $37^{\circ} \mathrm{C}$ in $5 \% \mathrm{CO}_{2}$ for 4 hours. The medium was aspirated, and $100 \mu \mathrm{L}$ of dimethyl sulfoxide (DMSO) was added to the wells. The plates were then stirred for $10 \mathrm{~min}$. After the crystals were completely dissolved, the OD values were measured at $490 \mathrm{~nm}$ using a microplate reader. Phosphate buffer saline (PBS) was as control and added in 96-well plates.

\subsection{Determination of ALP activity and $\mathrm{OCN}$}

MC3T3-E1 cells were inducted by induction medium with vitamin $\mathrm{C}(50 \mathrm{mg} / \mathrm{L})$ and $\beta$-sodium glycerophosphate $(10 \mathrm{mM})$ or uninduction medium without vitamin $C$ and $\beta$-sodium glycerophosphate, and LABS mixed at a ratio of $1: 20$ and $0.5 \mathrm{mM}$ sodium butyrate medium were collected after induction for 7 days, respectively. PBS was as control and added in plates. The media were centrifuged for $10 \mathrm{~min}$ at $1500 \mathrm{r} / \mathrm{min}$, and the supernatants were collected. The alkaline phosphatase (ALP) activity and 
osteocalcin $(\mathrm{OCN})$ concentration were measured by ELISA assay according to the manufacturer's instructions.

\subsection{Realtime PCR assay}

MC3T3-E1 and RAW 264.7 cells are treated in medium containing induction medium which MC3T3-E1 cells are inducted by vitamin $C(50 \mathrm{mg} / \mathrm{L})$ and $\beta$-sodium glycerophosphate $(10 \mathrm{mM})$, RAW264.7 cells are inducted by $50 \mu \mathrm{g} / \mathrm{L}$ RANKL, and LABS mixed at a ratio of $1: 20$ or $0.5 \mathrm{mM}$ sodium butyrate medium were collected. PBS was as control and added in plates. The total RNA was extracted using the TRIzol reagent (Invitrogen) according to the manual's instructions. Then cDNA was obtained by oligo-dT primers or stemloop reverse transcriptase (RT) primers by RT-PCR kit (Takara Bio Inc.), and mRNA expression of the target genes were detected using real-time PCR assay (Applied Biosystems 7500 Real-Time PCR). The RNA was used as a template for complementary DNA (CDNA) synthesis with the following conditions: denaturation at $95^{\circ} \mathrm{C} 10 \mathrm{~min}$, followed by 40 cycles at $95^{\circ} \mathrm{C} 15 \mathrm{~s}, 60^{\circ} \mathrm{C} 60 \mathrm{~s}, 60^{\circ} \mathrm{C} 15 \mathrm{~s}$ and a fnal elongation at $95^{\circ} \mathrm{C}$ for $15 \mathrm{~s}$. RT-qPCR was performed to detect wingless-type MMTV integration site family member 2 (WNT2), $\beta$ catenin gene (CTNNB1) and receptor activator for nuclear factor-K B (RANK), and GAPDH was used as the internal reference gene. The quantified results were calculated using the $2^{-\triangle \Delta C q}$ method (13). The full details of the primers used in these experiments are shown in Table 2

\subsection{Effects of bacterial supernatant on osteoblast and osteoclasts culturing and induction}

MC3T3-E1 cells are inducted by vitamin C $(50 \mathrm{mg} / \mathrm{L})$ and $\beta$-sodium glycerophosphate $(10 \mathrm{mM})$, RAW264.7 cells are inducted by $50 \mu \mathrm{g} / \mathrm{L}$ RANKL. Above induction medium are refreshed every 3 days.

The extracted LABS and LGG bacterial supernatant was mixed with at ratios of 1:20 to generate bacterial supernatant culture medium and $0.5 \mathrm{mM}$ sodium butyrate (represent butanoic acids) in which MC3T3-E1 and RAW 264.7 cells were incubated at $37{ }^{\circ} \mathrm{C}$ in $5 \% \mathrm{CO}_{2}$. The bacterial supernatant culture medium and sodium butyrate was replaced every 48 hours. PBS was as control and added in plates.

ALP/ Alizarin red staining was performed on the MC3T3-E1 cells after 7 days of incubation in induction medium. Two milliliters of citric acid concentrate was added to $100 \mathrm{ml}$ of deionized water and then mixed with acetone (2:3 ratio) to produce a fixing solution. The prepared fixing solution was added to the culture plates to fix the osteoblasts for $3 \mathrm{~min}$. Substrate was applied dropwise to completely cover the fixed osteoblasts, and the plates were incubated at $37^{\circ} \mathrm{C}$ for $15 \mathrm{~min}$ in the dark before being washed with distilled water for $5 \mathrm{~min}$. The cells were stained with Alizarin red solution for $7 \mathrm{~min}$ and then washed with distilled water for $5 \mathrm{~min}$. The slides were dried at room temperature and sealed. The cells were observed and photographed under a microscope. 
Tartrate-resistant acid phosphatase (TRAP) staining of RAW 264.7 osteoclasts was performed after treating them with the same method for 5 days.

\subsection{Statistical Analysis}

Data was represented by mean \pm SD and analyzed by SPSS 11.0 software. The one way ANOVA analysis tukey's posthoc was used for general measurement data. R software (Version, 2.15.3) was used to nonmetric multidimensional scaling (NMDS) analysis. $P<0.05$ was defined as a significant difference.

\section{Results}

\subsection{Comparison of the general data between the groups}

No statistically significant differences were observed between the two groups upon comparison of the biochemical parameters (ALB, ALT, AST, ALP, CREA, UREA, UA, FPG, Ca, P, K, Na, TG, TC, LDL and PTH) in Table 3, indicating that secondary osteoporosis caused by common reasons, such as endocrine diseases and nutritional factors, could be excluded. Moreover, the patients had no history of treatment with glucocorticoids, corticosteroids, antiepileptic drugs, antineoplastic drugs (e.g., methotrexate) or heparin. The levels of two bone turnover markers, 25-hydroxyvitamin D and PINP, in the osteoporosis group were significantly lower than those in the control group; however, there was no obvious abnormalities in the CTX and OCN between the two groups (Table 4). These features indicate a lack of active bone resorption in the osteoporotic patients with high-bone-turnover activity, thus demonstrating that the patients enrolled in the groups suffered from senile osteoporosis with low-bone-turnover activity. Therefore, these patients could be diagnosed with primary osteoporosis and met the criteria to be included in the subsequent experiments.

\subsection{Changes in the gut microbiota of osteoporosis patients}

High-throughput sequencing was used to analyze and compare the 16S rRNA sequences present in the gut microbiota of the osteoporosis patients and healthy controls. The structure and characteristic of the gut microbiota in the osteoporosis patients was altered (Fig.1). Especially, the levels of Lactobacillus and butyric acid-producing bacteria were decreased, and abundance of pathogenic bacteria, such as Clostridium, was increased. 


\subsection{Effects of LABS, LGG and sodium butyrate on the proliferation of osteoblasts and osteoclasts}

There is a significantly enhanced the proliferation of osteoblast MC3T3-E1 after incubation in LABS supernatant (1:20 and 1:50) for both 24 and $48 \mathrm{~h}$, meanwhile, LGG supernatant not enhanced the proliferation on the any ratio for $24 \mathrm{~h}$ and $48 \mathrm{~h}$. There is a significantly reduced the proliferation of osteoclast RAW 264.7 after incubation in LABS supernatant (1:20 and 1:50) for 24 and the ratio of 1:20, 1:50 and 1:100 for $48 \mathrm{~h}$, however, LGG supernatant could not reduced the proliferation by any ratio both for 24 and $48 \mathrm{~h}$. Sodium butyrate $(0.5 \mathrm{mM})$ could stimulate the proliferation of osteoblast MC3T3-E1 and significantly inhibit the proliferation of osteoclast RAW 264.7 for 24 and 48 h (Fig.2).

\subsection{Effects of LABS, LGG and sodium butyrate on differentiation related factors in osteoblasts and osteoclasts}

ALP and OC are markers of osteoblast differentiation and play important roles in bone formation. The higher the ALP activity and OCN expression, the higher the osteogenic activity of the osteoblasts (14).After 7 days, there was significantly higher ALP activity and OCN concentration by LABS (1:20), LGG (1:20) or $0.5 \mathrm{mM}$ sodium butyrate treatment of osteoblasts MC3T3-E1 in induction and uninduction medium containing, meanwhile, the ALP activity and OCN concentration was higher in induction compared to uninduction medium (Fig.3A).

Runt-related transcription factor 2 (RUNX2) is a specific osteoblast factor and is closely linked to osteoblast function. RANK plays an important role in bone resorption and is highly expressed on the surface of osteoclasts. It participates in osteoclast differentiation, thus leading to bone loss. The Wnt/ $\beta$ catenin signaling pathway stimulates osteoblasts and inhibits osteoclast. WNT2 encodes the wnt protein, and $\beta$-catenin is a protein encoded by the CTNNB1 gene. The mRNA expression of RUNX2, WNT2 and CTNNB1 was significantly increased in osteoblasts MC3T3-E1 incubated in media containing LABS (1:20) or $0.5 \mathrm{mM}$ sodium butyrate. The expression levels of the mRNAs of RANK, WNT2 and CTNNB1 significantly decreased in osteoclasts RAW 264.7 (Fig.3B).

\subsection{Effects of LABS, LGG and sodium butyrate on the maturity of osteoblasts and osteoclasts}

After ALP/Alizarin red staining, dark staining of the cell membrane and cytoplasm was observed. The results showed that the number of darkly stained cells (maturity osteoblasts) increased in the osteoblasts MC3T3-E1 cultured in media containing LABS (1:20) and sodium butyrate (0.5 mM). However, there was 
not significant increased by LGG (1:20). The MC3T3-E1 was cultured in induction medium as positive control (Fig.4A).

Following cultured with LABS (1:20), LGG (1:20) and sodium butyrate $(0.5 \mathrm{mM})$, the RAW 264.7 cells gradually differentiated into osteoclasts, i.e., osteoclasts gradually formed after 5 days of induction, as indicated by increased cytoplasmic volume and additional nuclei. The results showed that the number of darkly stained cells increased in osteoblasts cultured and induced in media containing LABS (1:20) or butyric acid (Fig.4B).

\section{Discussion}

The aging population and age-related bone damage have developed into major public health problems in China (15).Osteoporosis patients are not only increasing in number but also have increased risk of suffering from additional senile diseases (16). Osteoporotic fractures in these patients result in difficulties in treatment and care and can also cause many economic and social problems (17). Therefore, research on novel effective treatment methods and the mechanisms underlying senile osteoporosis is important, as it has practical and important implications.

Recent studies have found that the gut microbiota is associated with decreased bone mass and the pathogenesis of osteoporosis. These microorganisms may affect bone metabolism by altering the relative activities of osteoclasts and osteoblasts via different pathways, such as those involving bacterial metabolites and others affecting host metabolism and immunity (18-20). Although the gut microbiota varies widely, the composition of the gut microbiota in older people significantly shifts from obligate anaerobic bacteria to facultative anaerobic bacteria. In particular, the abundance of pathogenic proteobacteria increases, while that of anti-inflammatory lactobacilli decreases. These changes lead to increased risk of inflammation and, in turn, mediate the differentiation and maturation of osteoclasts. This process indicates that the characteristic changes in the gut microbiota of older people may increase their risk of osteoporosis. In this study, analyses of the fecal microflora from osteoporosis patients and healthy control subjects revealed dysbacteriosis, decreased levels of lactobacilli and some butyric acidproducing bacteria, and increased levels of pathogenic clostridium in the osteoporosis patients

Lactobacillus is one of the most widely studied probiotics, and the term "lactobacillus" is actually a generic name for any bacteria that can produce large amounts of lactic acid from fermentable carbohydrates (21).In this study, LABS and LGG, which belong to the genus Lactobacillus, were selected for study. The well-accepted roles of these two bacterial species include maintaining the balance of the gut microbiota, promoting defecation, reducing the occurrence of constipation, improving immunity, lowering blood lipid levels, promoting nutrient absorption, improving lactose intolerance and imparting anti-cancer effects. An increased proportion of probiotics, such as lactobacilli, can increase the levels of short-chain fatty acids, resulting in a positive effect on bone metabolism. Butyric acid, a short-chain fatty acid, can directly affect bone cells and inhibit the growth of osteoclasts in vitro and can stimulate the proliferation and differentiation of osteoblasts. 
The results of our experiments showed that the number of darkly stained cells increased in osteoblasts cultured and induced in media with LABS (1:20) or butyric acid. ALP/Alizarin red staining can make cell membrane and cytoplasm appear darkly stained. Therefore, this finding may indicate that the most mature and abundant osteoblasts were induced and differentiated in the LABS medium (1:20). This observation suggests that LABS metabolites may contain short-chain fatty acid that can directly stimulate osteoblasts. Furthermore, the levels of osteoblast stimulation were positively related to the metabolite concentration and the duration of incubation. In contrast, no significant increase in osteoblast activities was observed in the medium containing LGG supernatant, indicating that there were no shortchain fatty acids among its metabolites that could directly affect osteoblasts. It is known that LABS can produce butyric acid, but it is not clear whether LGG shares this trait. In light of the observation of the increased number of darkly stained osteoblasts after culturing and induction in medium containing 0.5 $\mathrm{mM}$ sodium butyrate, we conclude that LABS stimulates osteoblast proliferation, differentiation and maturation of via butyric acid in its metabolites, thereby improving bone quality.

Butyric acid, a short-chain fatty acid, induces cell morphology changes and cell differentiation in different cell types (22).However, the direct effects of this short-chain fatty acid on bone cells have not been well studied. In recent years, studies have shown that ALP and OCN are expressed throughout the processes of proliferation and differentiation in MC3T3-E1 cells. ALP and OCN are markers of osteoblast differentiation and play important roles in bone formation. It has been reported that butyric acid can increase ALP activity and OCN expression. This observation indicates that butyric acid can stimulate the activity of MC3T3-E1 cells to promote osteoblast proliferation and differentiation. This finding has been confirmed in this study. ALP activity and OCN expression were significantly increased after incubation with LABS medium (1:20) or $0.5 \mathrm{mM}$ sodium butyrate medium for 7 days, indicating that LABS promotes osteoblast differentiation and maturation via butyric acid production.

RUNX2 is associated with osteoblast differentiation. Its main function is to positively regulate osteoblast function and to stimulate ALP to promote bone maturation (23).RANK binds to the receptor activator for nuclear factor-K $B$ ligand (RANKL) released by osteoblasts. During bone resorption, osteoclast precursor cells constantly differentiate into osteoclasts, and RANK is highly expressed on the surface of the osteoclast precursor cells. RANK binds to the RANKL released by osteoblasts to produce downstream signals that increase the expression levels of many transcription factors in osteoclast precursor cells to activate the $\mathrm{Wnt} / \beta$-catenin pathway (24).The $\mathrm{Wnt} / \beta$-catenin pathway is an important signaling cascade that promotes osteoblast formation and inhibits osteoclast formation. Wnt protein and $\beta$-catenin are encoded by the WNT2 and CTNNB1 genes, respectively (25).In this study, treatment with LABS or butyric acid upregulated the mRNA expression levels of RUNX2, WNT2, CTNNB1 and RANK in MC3T3-E1 osteoblasts, and downregulated the expression levels of the above genes in RAW 264.7 osteoclasts. These results confirm that LABS and butyric acid can promote the differentiation and maturation of osteoblasts and inhibit the proliferation and differentiation of osteoclasts by regulating the expression levels of the above genes. 
The experimental results showed that a high concentration of LABS supernatant could stimulate the proliferation, differentiation and maturation of osteoblasts. The aforementioned effects were not present in cells exposed to LGG supernatant. LABS can produce butyric acid, which can stimulate osteoblast proliferation and differentiation, while LGG does not produce butyric acid. Based on these observations, we speculated that LABS may stimulate osteoblast proliferation, differentiation and maturation via butyric acid production.

\section{Declarations}

\section{Acknowledgements}

Not applicable.

\section{Funding}

This study was supported by National Natural Science Foundation of China (81570489), Scientific Research Project on Traditional Chinese Medicine/Integrative Medicine of the Tianjin Health and Family Planning Commission (Grant No. 2017084), the Special Program of Talent Development for Excellent Youth Scholars in Tianjin, China (grant no. TJTZJH-QNBJRC-2-9) and the Tianjin 131 Creative Talents Cultivation Project (1st Class, 2016).

\section{Availability of data and materials}

All data generated or analyzed during this study are included in this published article.

\section{Ethics approval and consent to participate}

Not applicable.

\section{Patient consent for publication}

Not applicable.

\section{Competing interests}

The authors declare that they have no competing interests. 


\section{References}

1. Li L, Rao S, Cheng Y, Zhuo X, Deng C, Xu N, Zhang H, Yang L.Microbial osteoporosis: The interplay between the gut microbiota and bones via host metabolism and immunity. Microbiologyopen. 2019; 18:e00810.

2. Yatsonsky li D, Pan K, Shendge VB, Liu J, Ebraheim NA.Linkage of microbiota and osteoporosis: A mini literature review. World J Orthop. 2019; 3:123-127.

3. Rizzoli R.Nutritional influence on bone: role of gut microbiota. Aging Clin Exp Res. 2019 Feb 1.

4. Shimizu Y.Gut microbiota in common elderly diseases affecting activities of daily living. World $J$ Gastroenterol. 2018; 42:4750-4758.

5. Quach D, Britton RA.Gut Microbiota and Bone Health. Adv Exp Med Biol. 2017;1033:47-58.

6. Brown EM, Kenny DJ, Xavier RJ.Gut Microbiota Regulation of T Cells During Inflammation and Autoimmunity. Annu Rev Immunol. 2019;37:599-624.

7. Pascale A, Marchesi N, Govoni S, Coppola A, Gazzaruso C. The role of gut microbiota in obesity, diabetes mellitus, and effect of metformin: new insights into old diseases. Curr Opin Pharmacol. 2019;49:1-5.

8. Laitinen K, Mokkala K.Overall Dietary Quality Relates to Gut Microbiota Diversity and Abundance. Int J Mol Sci. 2019; 8. pii: E1835.

9. Naderpoor N, Mousa A, Gomez-Arango LF, Barrett HL, Dekker Nitert M, de Courten B.Faecal Microbiota Are Related to Insulin Sensitivity and Secretion in Overweight or Obese Adults. J Clin Med. 2019; 4. pii: E452.

10. Cheng S, Han B, Ding M, Wen Y, Ma M, Zhang L, Qi X, Cheng B, Li P, Kafle OP, Liang X, Liu L, Du Y, Zhao Y, Zhang F.Identifying psychiatric disorder-associated gut microbiota using microbiota-related gene set enrichment analysis. Brief Bioinform. 2019; pii: bbz034.

11. Messina OD, Vidal Wilman M, Vidal Neira LF. Nutrition, osteoarthritis and cartilage metabolism. Aging Clin Exp Res. 2019 Apr 13.

12. Chen YC, Greenbaum J, Shen H, Deng HW.Association Between Gut Microbiota and Bone Health: Potential Mechanisms and Prospective. J Clin Endocrinol Metab. 2017; 10:3635-3646.

13. Livak KJ,Schmittgen TD. Analysis of relative gene expression data using real-time quantitative PCR and the 2(-Delta Delta C(T)) Methods. 2001; 4:402-8.

14. Liu L, Wang D, Qin Y, Xu M, Zhou L, Xu W, Liu X, Ye L, Yue S, Zheng Q, Li D.Astragalin Promotes Osteoblastic Differentiation in MC3T3-E1 Cells and Bone Formation in vivo. Front Endocrinol (Lausanne). 2019;10:228.

15. Sun R, Cao H, Zhu X, Liu JP, Dong E.Current aging research in China.Protein Cell. 2015; 5:314-21.

16. Tian L, Yang R, Wei L, Liu J, Yang Y, Shao F, Ma W, Li T, Wang Y, Guo T.Prevalence of osteoporosis and related lifestyle and metabolic factors of postmenopausal women and elderly men: A crosssectional study in Gansu province, Northwestern of China.Medicine (Baltimore). 2017; 43:e8294. 
17. Tao L, Huang Q, Yang R, Dai Y, Zeng Y, Li C, Li X, Zeng J, Wang Q.The age modification to leukocyte telomere length effect on bone mineral density and osteoporosis among Chinese elderly women. $J$ Bone Miner Metab. 2019 Apr 20.

18. Liang M, Zhang B, Deng L, Xu R, Wu H, Chen J.Effects of Olanzapine on Bone Mineral Density, Glucose, and Lipid Metabolism in Schizophrenia Patients. Int J Endocrinol. 2019;2019:1312804.

19. Zhao W, Zhang WL, Yang B, Sun J, Yang MW.NIPA2 regulates osteoblast function via its effect on apoptosis pathways in type 2 diabetes osteoporosis. Biochem Biophys Res Commun. 2019; pii: S0006-291X(19)30652-7.

20. Coury F, Peyruchaud O, Machuca-Gayet I.Osteoimmunology of Bone Loss in Inflammatory Rheumatic Diseases. Front Immunol. 2019;10:679.

21. Duar RM, Lin XB, Zheng J, Martino ME, Grenier T, Pérez-Muñoz ME, Leulier F, Gänzle M, Walter J.Lifestyles in transition: evolution and natural history of the genus Lactobacillus. FEMS Microbiol Rev. 2017; Supp_1:S27-S48.

22. Choi SI, Kim N, Lee SM, Nam RH, Kang SR, Song CH, Park YT, Min H, Kim YR, Seok YJ.Rat Intestinal Acetic Acid and Butyric acid and Effects of Age, Sex, and High-fat Diet on the Intestinal Levels in Rats. J Cancer Prev. 2019; 1:20-25.

23. Wang R, Xu X, Li Y, Li J, Yao C, Wu R, Jiang Q, Shi D.A C-Met chemical inhibitor promotes fracture healing through interacting with osteogenic differentiation via the mTORC1 pathway. Exp Cell Res. 2019; pii: S0014-4827(19)30147-8.

24. Liang M, Ma Q, Ding N, Luo F, Bai Y, Kang F, Gong X, Dong R, Dai J, Dai Q, Dou C, Dong S.IL-11 is essential in promoting osteolysis in breast cancer bone metastasis via RANKL-independent activation of osteoclastogenesis. Cell Death Dis. 2019; 5:353.

25. Xi FX, Wei CS, Xu YT, Ma L, He YL, Shi XE, Yang GS, Yu TY.MicroRNA-214-3p Targeting Ctnnb1 Promotes 3T3-L1 Preadipocyte Differentiation by Interfering with the Wnt/ $\beta$-Catenin Signaling Pathway. Int J Mol Sci. 2019; 8. pii: E1816.

\section{Tables}

Table 1 The general data of normal and osteoporosis group

\begin{tabular}{ccccc}
\hline & $\begin{array}{c}\text { Age } \\
\text { (years) }\end{array}$ & systolic pressure $(\mathrm{mmHg})$ & diastolic pressure $(\mathrm{mmHg})$ & $\begin{array}{c}\text { BMI } \\
\left(\mathrm{kg} / \mathrm{m}^{2}\right)\end{array}$ \\
\hline Control & $90.14 \pm 6.07$ & $128.86 \pm 16.87$ & $61.86 \pm 8.51$ & $22.47 \pm 2.40$ \\
osteoporosis & $88.50 \pm 5.40$ & $129.25 \pm 22.05$ & $60.13 \pm 9.37$ & $23.79 \pm 2.19$ \\
\hline$P$ value & & $>0.05$ & \\
\hline
\end{tabular}

Table 2 Quantitative real time polymerase chain reaction primers in the experiments 


\begin{tabular}{cc}
\hline Gene & \multicolumn{1}{c}{ Primers } \\
\hline WNT2 & Forward: 5'- CTCGGTGGAATCTGGCTCTG -3' \\
& Reverse: 5'- CACATTGTCACACATCACCCT -3' \\
RANK & Forward: 5'- CTCTATGCCCGTGTCCCCTGAAAA -3' \\
& Reverse: 5'- GGCCGCGATGTCCCGTCCTT -3' \\
CTNNB1 & Forward: 5'-ATGGAGCCGGACAGAAAAGC-3' \\
& Reverse: 5'-CTTGCCACTCAGGGAAGGA-3' \\
GAPDH & Forward: 5'- AGGTCGGTGTGAACGGATTTG -3' \\
& Reverse: 5'- TGTAGACCATGTAGTTGAGGTCA -3' \\
\hline
\end{tabular}

Table 3 The clinic biochemistry data of normal and osteoporosis group

\begin{tabular}{llll}
\hline & control & osteoporosis & $P$ value \\
\hline TP $(\mathrm{g} / \mathrm{L})$ & $67.43 \pm 6.13$ & $65.75 \pm 3.85$ & $>0.05$ \\
ALB $(\mathrm{g} / \mathrm{L})$ & $34.57 \pm 2.94$ & $34.25 \pm 2.66$ & \\
ALT (U/L) & $11.57 \pm 3.46$ & $15.63 \pm 8.91$ & \\
AST (U/L) & $17.57 \pm 4.79$ & $18.88 \pm 8.08$ & \\
ALP (U/L) & $74.14 \pm 7.65$ & $77.13 \pm 44.08$ & \\
TBIL $(\mu M)$ & $8.57 \pm 3.22$ & $8.05 \pm 2.35$ & \\
UREA (mM) & $5.43 \pm 2.74$ & $7.49 \pm 6.01$ & \\
CREA ( $\mu M)$ & $70.43 \pm 15.60$ & $69.13 \pm 36.37$ & \\
URIC $(\mu M)$ & $281.71 \pm 75.60$ & $271.86 \pm 123.11$ & \\
GLU (mM) & $4.97 \pm 0.53$ & $5.03 \pm 0.58$ & \\
Ca (mM) & $2.26 \pm 0.96$ & $2.30 \pm 0.15$ & \\
PHOS (mM) & $0.98 \pm 0.15$ & $1.00 \pm 0.11$ & \\
K (mM) & $4.31 \pm 0.56$ & $4.13 \pm 0.39$ & \\
Na (mM) & $140.86 \pm 1.35$ & $139.63 \pm 2.77$ & \\
TC (mM) & $4.32 \pm 0.77$ & $4.66 \pm 1.09$ & \\
TG (mM) & $1.32 \pm 0.33$ & $1.65 \pm 0.70$ \\
LDL (mM) & $2.86 \pm 0.65$ & $2.94 \pm 0.77$ \\
PTH (pM) & $7.20 \pm 3.19$ & $5.24 \pm 2.70$ & \\
\hline
\end{tabular}

Table 4 The Vitamin D and bone turnover marker of normal and osteoporosis group

\begin{tabular}{ccccc}
\hline & $\begin{array}{c}25-\mathrm{OH} \text { VitaminD } \\
(\mathrm{nM})\end{array}$ & $\begin{array}{c}\text { OC } \\
(\mathrm{ng} / \mathrm{ml})\end{array}$ & $\begin{array}{c}\text { CTX } \\
(\mathrm{ng} / \mathrm{ml})\end{array}$ & $\begin{array}{c}\text { PINP } \\
(\mathrm{ng} / \mathrm{ml})\end{array}$ \\
\hline Control & $50.17 \pm 13.59$ & $21.36 \pm 7.13$ & $0.47 \pm 0.21$ & $78.78 \pm 34.22$ \\
osteoporosis & $26.51 \pm 11.39$ & $19.6 \pm 4.59$ & $0.68 \pm 0.27$ & $41.83 \pm 13.16$ \\
\hline$P$ value & $<0.05$ & $>0.05$ & $>0.05$ & $<0.05$ \\
\hline
\end{tabular}

\section{Figures}


A

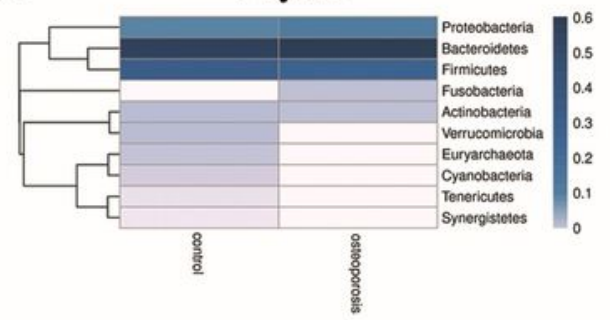

Family

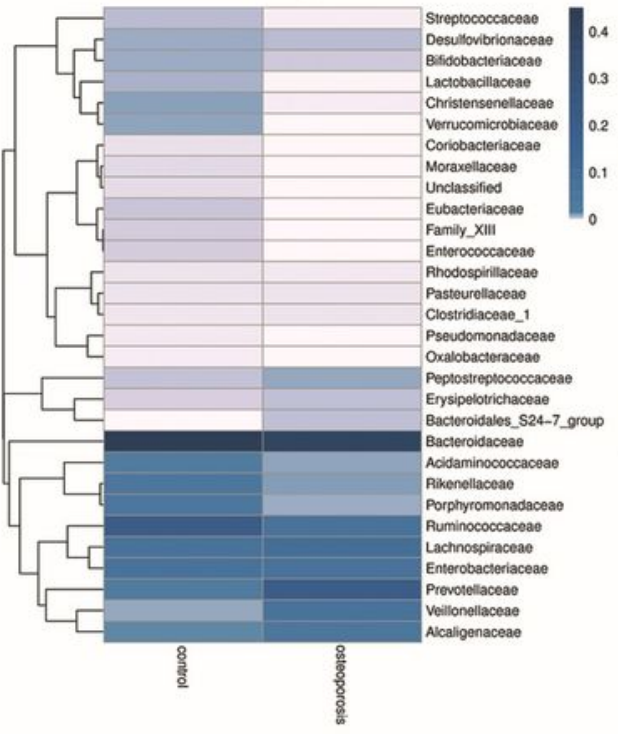

C

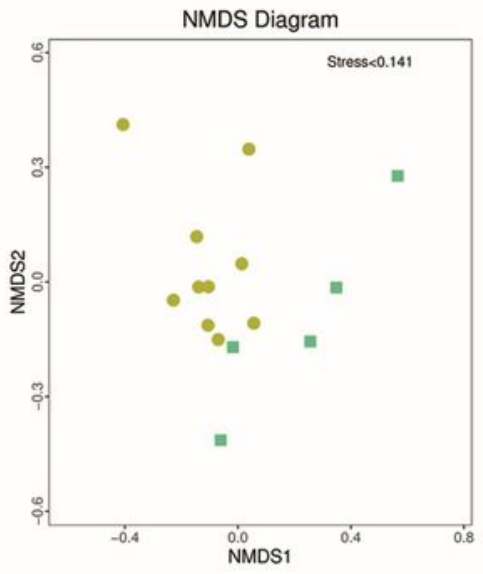

Class

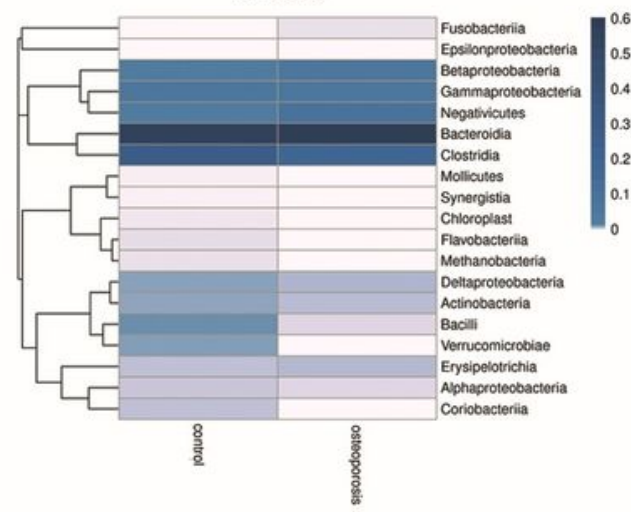

Genus

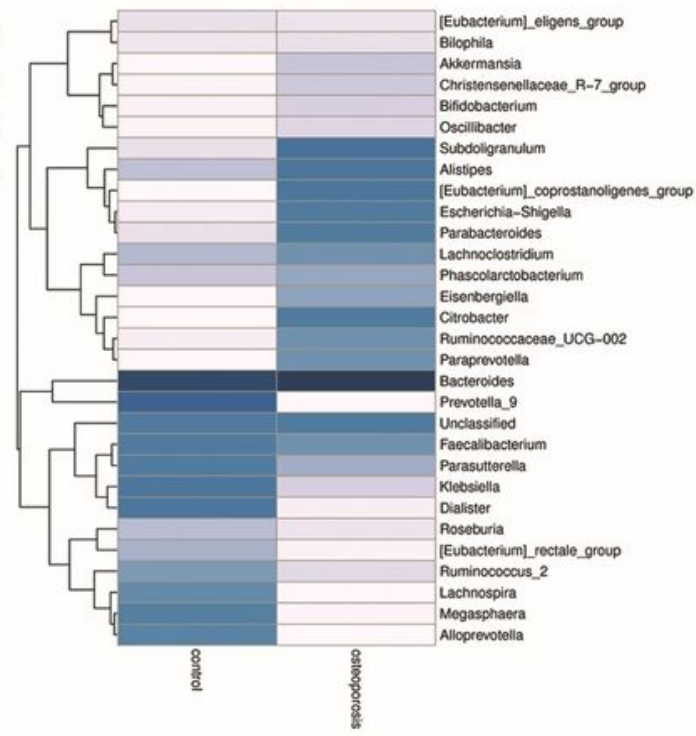

D

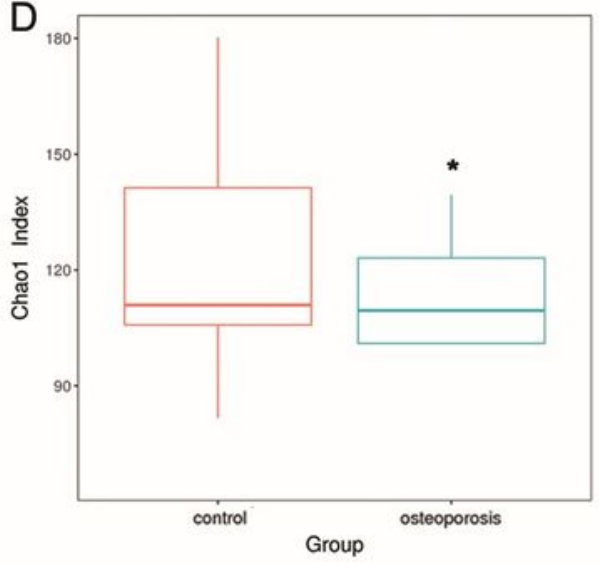

Order

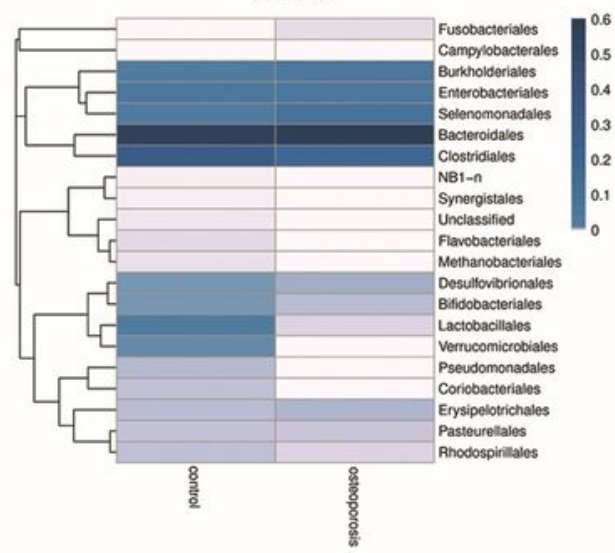

B

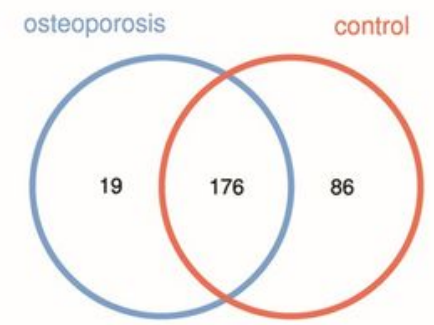

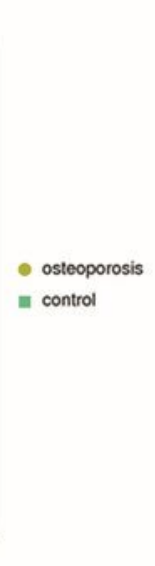

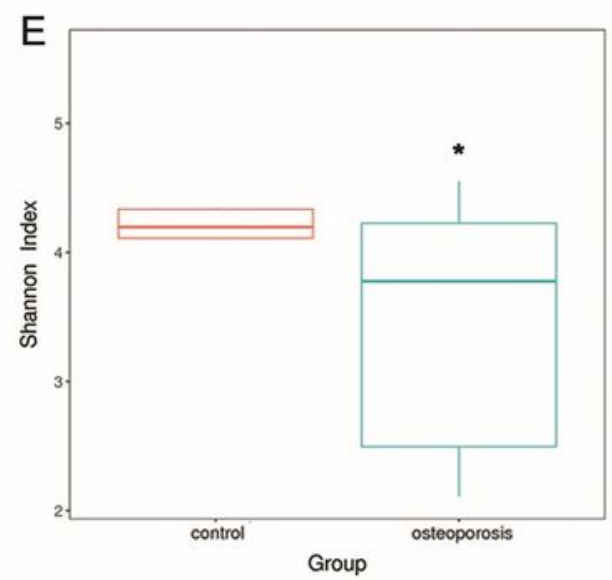

\section{Figure 1}

The characteristics of gut microbiota of healthy controls and osteoporosis patients. (A) The head map of gut microbiota of healthy controls and osteoporosis patients in different classification. (B) Venn diagram show that there are 86 changed OTU in healthy controls and 19 changed OTU in osteoporosis patients gut microbiota. (C) The NMDS analysis show that there is a heterogeneity of intestinal microbiota in the healthy controls and osteoporosis patients. (D) The Chao1 index and (E) Shannon index show that there 
is a decreased diversity in intestinal microbiota of osteoporosis patients compared with healthy control. *, compared with control group, $\mathrm{P}<0.05$.
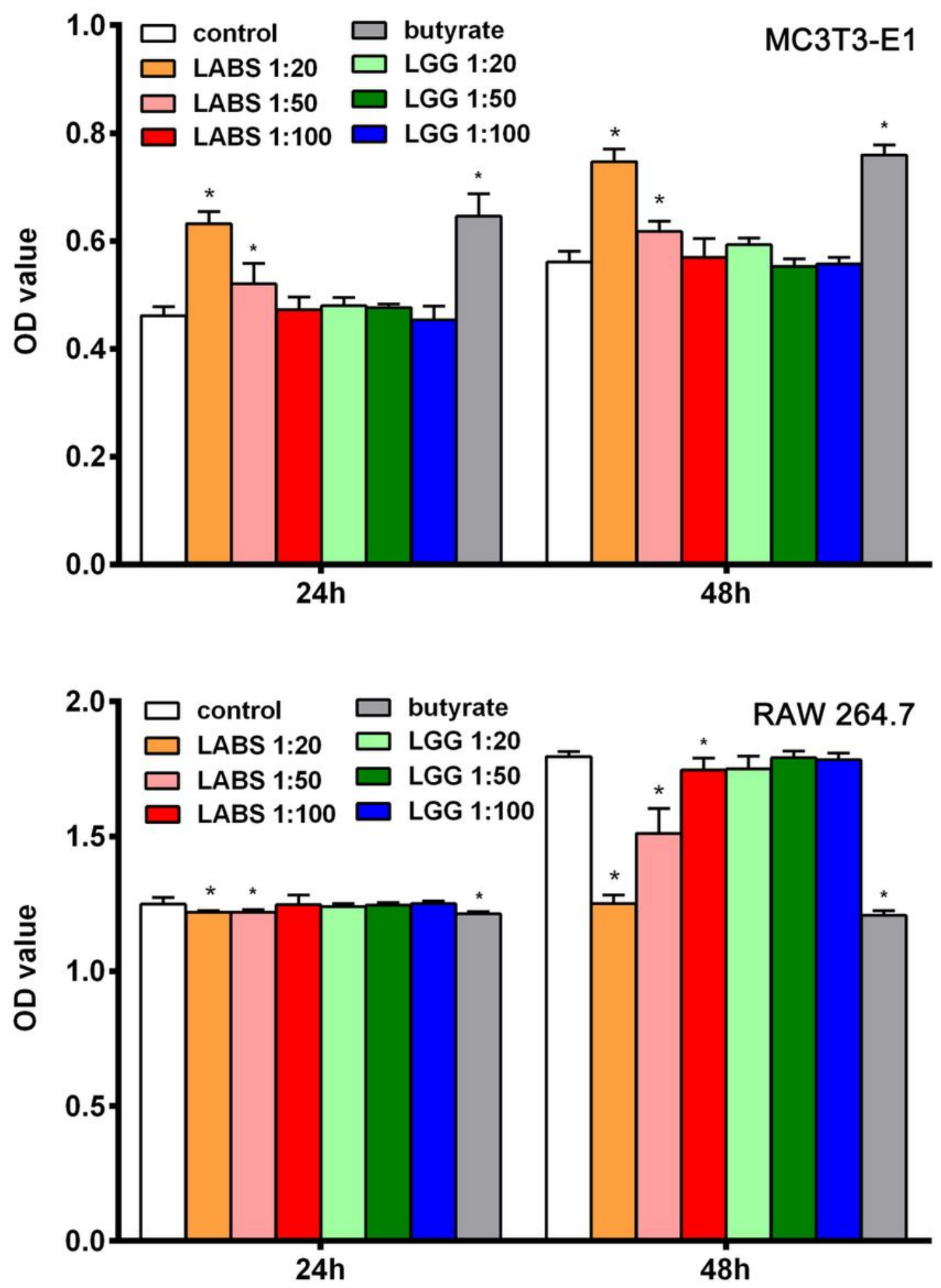

Figure 2

Effects of LABS, LGG and sodium butyrate on proliferation in osteoblasts and osteoclasts. MTT assay results showed that LABS supernatant (1:20 and 1:50) and sodium butyrate $(0.5 \mathrm{mM})$ enhanced the proliferation of osteoblast MC3T3-E1 for both 24 and 48 h, meanwhile, LGG supernatant could not 
enhanced the proliferation by any ratio both for 24 and $48 \mathrm{~h}$. LABS supernatant (1:20 and 1:50) and sodium butyrate $(0.5 \mathrm{mM})$ for both 24 and $48 \mathrm{~h}$, and LABS supernatant $(1: 100)$ for $48 \mathrm{~h}$, reduced the proliferation of osteoblast RAW 264.7. LGG supernatant could not reduced the proliferation by any ratio both for 24 and 48 h. *, P<0.05 compared with control group.
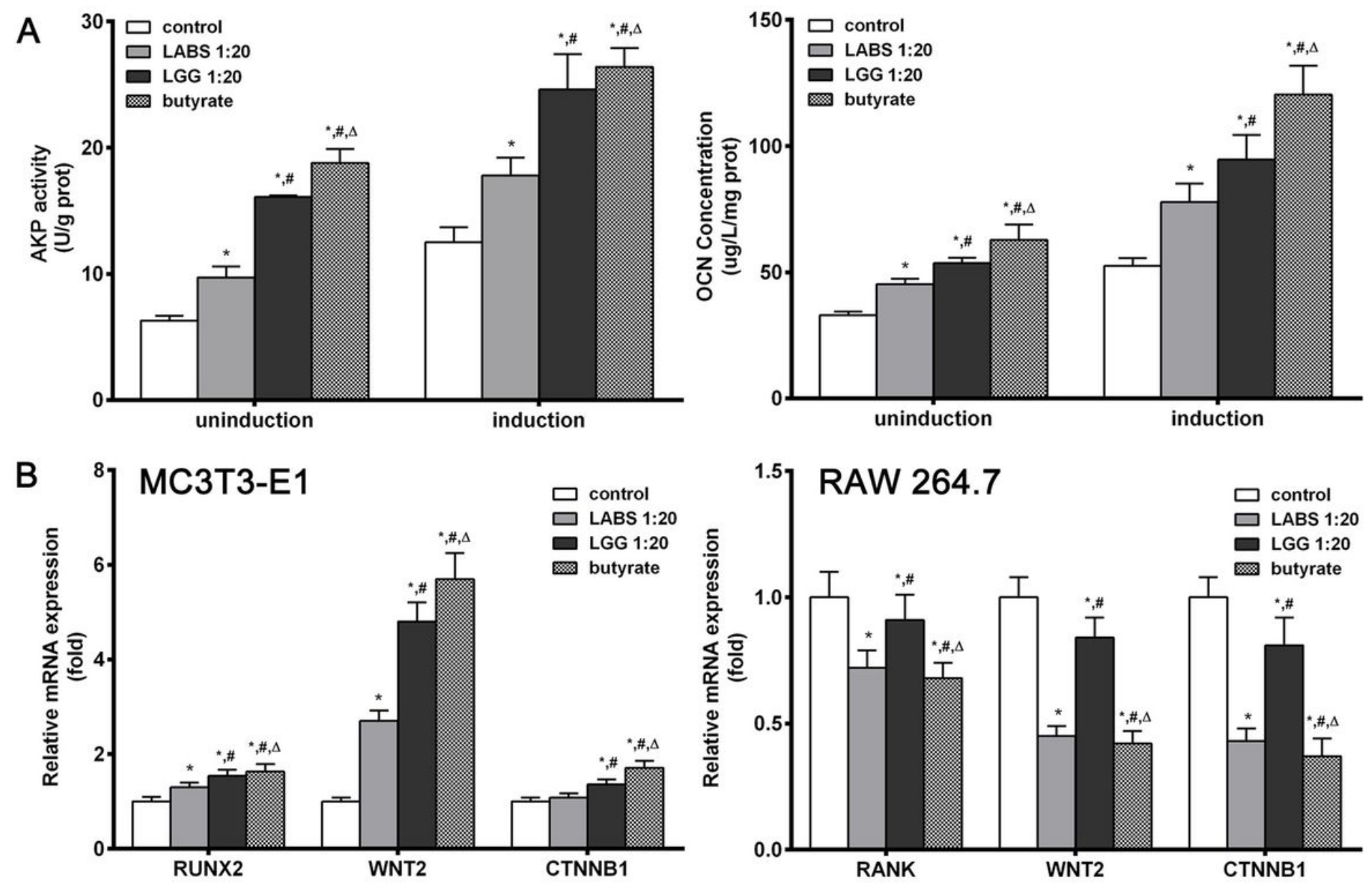

Figure 3

Effects of LABS, LGG and sodium butyrate on differentiation related factors in osteoblasts and osteoclasts. (A) The effect of LABS, LGG and sodium butyrate on ALP activity and OCN concentration in osteoblasts MC3T3-E1. (B) The effect of LABS, LGG and sodium butyrate on the expression of osteoblast and osteoclast related gene in osteoblasts MC3T3-E1 and osteoclasts RAW 264.7. *, $P<0.05$ compared with control group; \#, $P<0.05$ compared with LABS group; $\triangle, P<0.05$ compared with LGG group. 
A
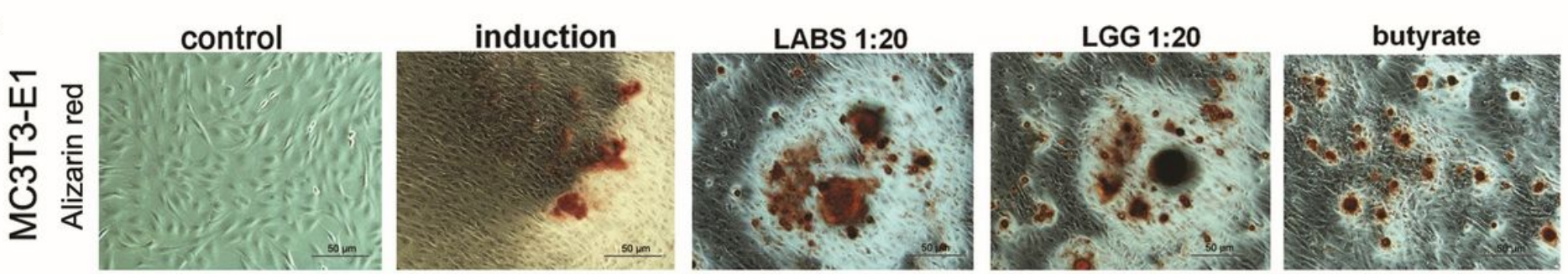

control
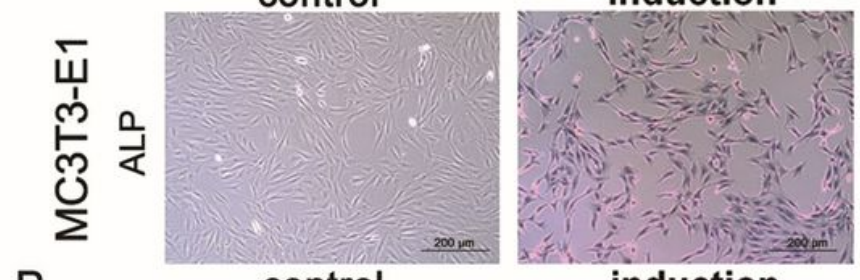

LABS 1:20

LGG 1:20
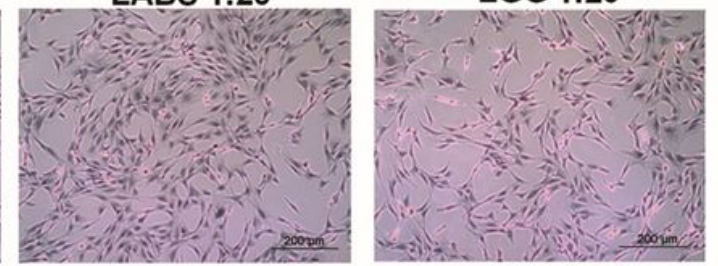

LABS 1:20
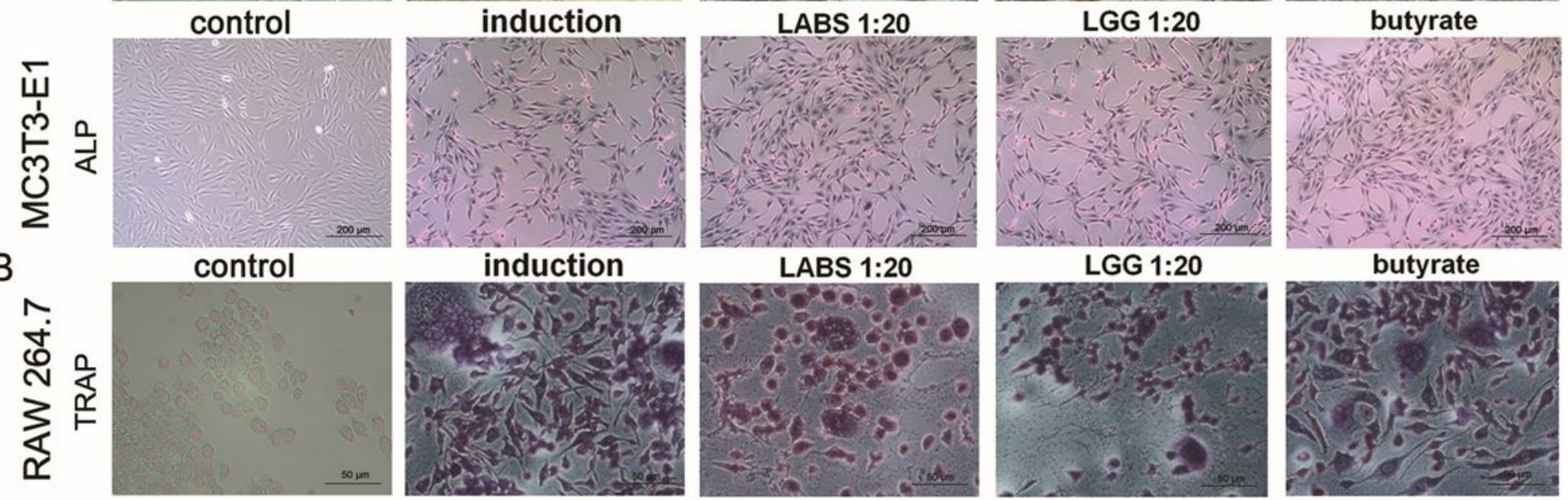

butyrate

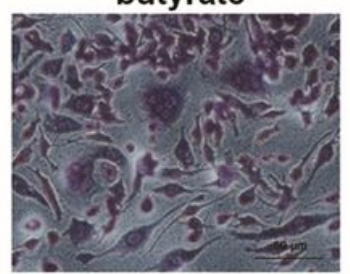

Figure 4

Effects of LABS, LGG and sodium butyrate on the maturity of osteoblasts and osteoclasts. (A) The effect of LABS, LGG and sodium butyrate on dark staining of the osteoblasts MC3T3-E1 cell membrane and cytoplasm after ALP/Alizarin red staining. (B) The effect of LABS, LGG and sodium butyrate on the cytoplasmic volume and additional nuclei in osteoclasts RAW 264.7 after TRAP staining. 